\title{
Large Time Stability Control for a Class of Quasilinear Parabolic and Hyperbolic Equations
}

\author{
Hongen $\mathrm{Li}^{1, \mathrm{a}}$, Shuxian Deng ${ }^{1,2, \mathrm{~b}}$ \\ ${ }^{1}$ Department of Basic Courses, Zhengzhou University of Industrial Technology, Xinzheng, China \\ ${ }^{2}$ College of Science, Henan University of Engineering, Zhengzhou, China \\ aemail: 857931663@qq.com, bemail:dshuxian@163.com
}

Keywords: Large; Decay; Inequality; Time.

\begin{abstract}
In this paper, we concerned with the stability control of solutions for a class of quasi-linear wave equations with weak attenuation. We use a so-called energy perturbation method to establish weak controllability of solutions in terms of energy norm for a class of nonlinear functions. We show the weak controllability with the help of differential inequalities by estimating the relationship between energy inequalities and attenuating property of weak solutions.
\end{abstract}

\section{Introduction}

We consider the control problem of weak decay to the following so-called quasi-linear wave equation in a smooth bounded domain $\Omega \subset \mathrm{R}^{3}$

$$
\begin{aligned}
& u_{t t}-\lambda \Delta u_{t}+u \cdot \nabla u=f(x, t) \\
& \operatorname{div} u=0 \\
& \left.u\right|_{\partial \Omega}=0 \\
& u(x, 0)=u_{0}, \quad u_{t}(x, 0)=u_{1}
\end{aligned}
$$

where $u_{0}, u_{1}, f$ are given functions, $\Delta u$ is a Laplacian with respect to the variable $x \in \Omega, u=u(t, x)$ is an unknown function, $\lambda>0$ is a fixed positive number, $f \in L^{2}(\Omega)$ are given external forces, and satisfying the following conditions:

$$
f \in L_{2}, u \in L_{q}\left(R^{+}, L_{p}\right) \text { and } \frac{2}{p}+\frac{n}{q} \leq 1
$$

For some positive $a, p \in[1 / 2,2), C>0$, and $q>0$, we have a weak solution, which fulfills additionally

$$
d+a|x|^{p} \leq f^{\prime}(x, t) \leq d\left(1+|x|^{q}\right), \forall s \in R^{3}
$$

And

$$
u_{t t}-\operatorname{div} \lambda \nabla u^{2}-\Delta u_{t}=f(x, t)
$$

We have several techniques to prove the existence of weak decay solutions with respect to the phase space $X^{\ell} \times X^{\tau}, X^{\varepsilon}:=D\left(\Omega^{q / p}\right)$, and have additional nice properties with energy inequality for almost all times or solutions with weak decay properties for $t \rightarrow \infty$, this has been studied recently by several people, e.g. Y.M. Qin, Ebihara, Xin Liu [1-6] etc.

As a model of the quasi-linear equation, for $N=1 / 2,1$ and $f=0,1$ equation (1.1)-(1.4) admits a global weak decay solution as large initial data, which was proved by Y.M. Qin, Xin Liu, X.G. Yang, Lan Huang etc [2-3]. TG Wang, Ming Zhang, M.J. Wang simplified the above arguments and give the proof of control with exponential decay. From the perspective and background of physics, this represents an implementation of an axial movement of the viscoelastic material, this causes the form of above equations, in the one-dimensional case, their model of longitudinal vibration of a uniform rod with nonlinear stress function $f$. In two, three-dimensional case, they describe the viscoelastic solid of anti-plane shear action. While $n=1$ and $f=0$, S.T. Li prove the existence of 
weak periodic decay strong solution on the periodicity condition, X.K. Su and JL Zhang[3-6] proved the controllability of a smooth solution in the method of Cauchy problems in the case of smooth and small data.

Ulteriorly, while $n>1 / 2$ and $f \neq 0$, M.J. Wang and X.G. Yang gave the proof of global controllability with a smooth solution in the case of small initial data. Make use of combining $L^{p}$-theorem of Sobolev space and semigroup theorem of operators, Nakao [4] and T. Kato [5] devised certain decay rate of the energy of global solutions with large data under a specific condition which is certainly satisfied if the mean curvature of the boundary $\partial \Omega$ is non-positive. For $n>1$ and $f=1$, nonlinear elliptic equation with periodicity conditions was studied [10],

$$
\begin{gathered}
d^{\prime}(t)+k_{1}\left\|w_{1}(t)-w_{2}(t)\right\|_{p}^{D} d(t) \leq k_{2} d(t)^{p+q-1}+\kappa d(t)^{\mu}\left\|w_{1}(t)\right\|_{2}^{v+1} \\
\left\|w(t)-w_{0}(t)\right\|_{p} \leq k(\lambda+x)^{\frac{d}{2}-\frac{n}{2}\left(\frac{\kappa}{2}-\frac{1}{p}\right)} \\
w(x, t+\omega)=w(x, t) \\
\left.w\right|_{\partial}=0
\end{gathered}
$$

In the case of $\|w(t)\| \leq k_{0}(1+t)^{-\frac{\lambda}{2}}$, and $\|w(t)\|_{p} \leq \tau(1+t)^{\left(\frac{\tau}{2}+\frac{n}{2}\left(\frac{1}{2}-\frac{1}{p}\right)\right)}$. Here, $\Omega$ is a bounded domain in $R^{n}$ with a smooth, $\partial \Omega$ is said to be $C^{2}$ class boundary, which satisfies the following uniform hyperbolic assumption:

For some constants $\rho_{0}, M>0, \tau \in H^{4}[0,+\infty)$ satisfies:

$$
x^{\prime}(t)+\tau_{0}(1+t)^{\left(\ell+\frac{d}{2}\right)} x(t)^{\left(2+\frac{\gamma}{p}\right)} \leq \sigma y(t)^{\frac{v}{2}}+\tau_{1}(1+t)^{\delta} y(t)^{\gamma}
$$

With $w(t)=u(t)-u_{0}(t), y(t)=\|w(t)\|_{p}^{m}$ and

$$
\begin{gathered}
\left\|w_{t}\right\|-\Delta w+\|u(r)\|_{q}=\frac{n}{2}+1-p n+2 p \delta \\
\tau\left(v^{2}\right) \geq \sigma_{0}>0 \text { and } \tau\left(v^{2}\right)+2 \sigma_{0}(v) v^{2} \geq \delta_{0}>0 \\
\tau\left(v^{2}\right)+2 \sigma_{0}\left(v^{2}\right) v+\tau^{\prime \prime}\left(v^{2}\right) \leq M<\infty
\end{gathered}
$$

Thus, we can discuss and conclude some results with lemmas in the next text[4-6].

\section{Preliminary Knowledge}

Lemma 1. Let $f(x, t) \in H^{1}\left([0, \omega], L^{2}()\right)$, and then with the above assumption, the problem admits a $\omega$-periodic solution $u(t)$ and the following estimates hold

$$
\begin{gathered}
\sup _{t \in[0, \omega]}\left\{\|u(t)\|^{2}+\left\|\nabla u_{t}(t)\right\|^{2}\right\}+\int_{0}^{\omega}\left\|u_{t}(s)\right\|^{2} d s \leq C M_{0}^{2} \\
\sup _{t \in[0, \omega]}\left\{\left\|u_{t}(t)\right\|^{2}+\left\|\nabla u_{t t}(t)\right\|^{2}\right\}+\int_{0}^{\omega}\left\|u_{t t}(s)\right\|^{2} d s \leq C\left(M_{0}^{2}+M_{1}^{2}\right) \\
M_{i}^{2}=\int_{0}^{\omega}\left\|\frac{\partial^{i}}{\partial t^{i}} f(s)\right\| d s, i=0,1
\end{gathered}
$$

Where

Lemma 2. Let $u_{0}, u_{1} \in H^{2}(\Omega) \cap H_{0}^{1}(\Omega)$ and $f(x, t) \in H^{1}\left(I, L^{2}()\right)$, then under assumption $H^{1}$, the problem admits a unique solution $u(t)$ and furthermore, if $\|f(t)\|^{2}+\left\|f_{t}(t)\right\|^{2}=O\left(e^{-\alpha t}\right)$ for some constant $\alpha>0$ as $t \rightarrow \infty$, the estimate 


$$
\left\|u_{t t}\right\|^{2}+\|\Delta u\|^{2}+\left\|\nabla u_{t}(t)\right\|^{2}+\left\|\Delta u_{t}\right\|^{2}+\int_{t}^{\infty}\left\|\nabla u_{t t}\right\|^{2} d s \leq C e^{-\beta t}
$$

Holds [3] for some constant $\beta>0$, where $C>0$ is a constant depending on $\left\|u_{0}\right\|_{H^{2}}+\left\|u_{1}\right\|_{H^{2}}$.

It turns out that this has an influence on the control of weak solution with structural damping. We can continue the following argument.

\section{Main Results}

We focus on the control of weak decay stability in (1.1)-(1.4), in order to describe the maneuverability; let us define global weak solution with decay for equations (1.1)-(1.4):

If the initial data satisfies $u_{0}, u_{1} \in H^{2} \cap H_{0}^{4}$, construct a weak solution space with

$$
\Theta=\left\{\Gamma: \times\left. w_{1 / 2}^{2} \rightarrow R\right|_{\Gamma} \in L^{\infty}\left(w_{1 / 2}^{2}, H^{2}() \cap H_{0}^{4}()\right), \Gamma^{\prime} \in L^{\infty}\left(w_{1 / 2}^{2}, H_{0}^{4}()\right)\right\}
$$

The function $\omega$ is said to be a weak solution of Problem (1.1)-(1.4), if it satisfies the following conditions:

(a) $\omega \in \Theta$

(b) $E(t) \equiv \frac{1}{2}\left\{\left\|\omega_{t}(t)\right\|^{2}+\int_{\Omega} \int_{0}^{|\nabla \omega|^{2}} \tau(\xi) d \xi d y\right\}$

$$
=\int_{0}^{t}(f, \omega(t)) d s d y-\int_{0}^{t}\left\|\nabla \omega_{t}(t)\right\|^{2} d s+\frac{1}{2}\left\{\left\|\nabla \omega_{1}(t)\right\|^{2}+\int_{\Omega} \int_{0}^{\left|\nabla \omega_{0}\right|^{2}} \tau(\xi) d \xi d y\right\}
$$

(c) $\left.\left(\omega_{t}, \mu\right)\right|_{0} ^{t}+\int_{0}^{t}\left\{\left(\omega_{t}, \mu_{t}\right)-(\operatorname{div}(\tau \nabla \omega), \mu)-\left(\Delta \omega_{t}, \mu\right)-(f, \mu)\right\}=0$

It is well known that the existence of such a weak solution with decay for all times is assured. Once this is known, one can identify this solution with the global weak one and continue this process to get that

$$
\left(\partial_{t}^{2} u, \phi\right)=\rho\left(\partial_{t} v, \Delta_{x} \phi\right)+\left(v, \Delta_{x} \phi\right)-\left(\varphi^{\prime}\left(\nabla_{x} \omega\right), \nabla x \phi\right)-(f(u), \phi)+(g, \phi)
$$

And

$$
t^{2}\left\|\partial_{t} v\right\|_{H}^{2} \leq \lambda t^{2}\left\|\partial_{t} v\right\|_{L^{\infty}}^{1 / 2}\left\|\partial_{t} v\right\|_{L^{2}}^{3 / 2} \leq \delta t^{3}\left\|\partial_{t} v\right\|_{L^{2}}^{3}+C^{3} \rho^{2} \delta^{-2}\left\|\partial_{t} \omega\right\|_{L^{\infty}}^{2}
$$

Obviously, we will construct the controllability of the local weak solution with decay for the semi-group generated by weak energy solutions.

Theorem 1. Let $\omega$ be a weak solution with the decay of (1.1)-(1.4). Denote by $\omega_{0}(t)=e^{-t \Delta}$ the solution of the wave equation and suppose $\left\|\omega_{0}(t)\right\|_{2} \leq \lambda_{0}(1+t)^{-\tau / 2}, \omega_{0} \in H^{2}() \cap H_{0}^{4}()$, $\omega_{1} \in H_{0}^{4}(), \quad f(x, t) \in H_{0}^{2}\left(\Theta, L^{2}()\right)$ and $f^{\prime}(x, t) \in W_{0}^{2}\left(\Theta, L^{2}()\right)$, then problem(1.1)-(1.4) hold a unique local weak solution $\omega(t)$ with the following estimate:

$$
\begin{gathered}
y^{\prime}(t)+\lambda_{0}(1+t)^{\frac{\kappa}{p}} y(t)^{\left(1+\frac{\varepsilon}{2} \kappa\right)} \leq \lambda_{1} y(t)^{\frac{\gamma}{2} \mu}+\lambda_{2}(1+t)^{-q} y(t)^{\sigma} \cdot \nabla x \omega(t) \\
\omega \in L^{\infty}\left([0, T], H^{2}() \cap H_{0}^{4}()\right), \omega^{\prime} \in L^{\infty}\left(\Theta, H_{0}^{4}()\right), \omega^{\prime \prime} \in W_{0}^{2}\left(\Theta, L^{2}()\right)
\end{gathered}
$$

\section{Conclusions}

In this section, we will draw our conclusions by means of some methods in the preliminary knowledge.

In fact, for $n=3 \omega \in L_{1} \cap L_{p}$, and $\left\|v(t)-v_{0}(t)\right\| \leq \sigma(1+t)^{\frac{\gamma}{3}\left(1-\frac{1}{q}\right)}$ note that the initial data is dense, hence $\left\|v_{0}(t)\right\|$ attenuates exponentially fast, Indeed, using $\omega|\omega|^{\frac{2 p}{n(p-2)}}$ and $\partial_{t} \omega$ multiply the equation (1.7) and integrate by parts over $x \in \Omega$. One gets 


$$
\begin{gathered}
\frac{\partial}{\partial t}\left(\left\|\partial_{t}(\omega)\right\|+\lambda(\theta(\nabla u), 1)+\sigma\|\nabla \omega\|_{L^{\infty}}^{2}+\frac{1}{2}(G(v), 1)+(\varphi, \varepsilon)\right)+\eta\left\|\nabla \partial_{t} h\right\|_{H_{0}^{2}}^{2}=0 \\
\delta^{\prime}(t)+\lambda_{1}(1+t)^{\kappa \frac{d}{2}} y(t)^{\lambda+\frac{\kappa}{p}} \leq \lambda_{2} \delta(t)^{\frac{n}{2}\left(\frac{1}{r}-\frac{1}{2}\right)}+\varepsilon(1+t)^{-(p-r)} \delta(t)^{\frac{1}{q}}
\end{gathered}
$$

Where $\varepsilon$ is a small positive number which will be fixed, Then we arrive at

$$
\begin{aligned}
\frac{\partial}{\partial t}\left(\delta \frac{\rho}{2}\left\|\partial_{t}(\omega)\right\|^{2}+\lambda\left(\partial_{t}(\omega), \omega\right)\right)-\sigma\left\|\partial_{t} \omega\right\|_{L^{\infty}}^{2}+\theta\|(\nabla u)\|^{2} \\
+\tau\left(\varphi^{\prime}(\nabla \omega), \nabla \omega\right)+\frac{1}{2} v(G(v), v)=\beta(g, \omega)
\end{aligned}
$$

And notice that

$$
E\left(\xi_{u}(t)\right)=\frac{p-2}{p}\left\|\partial_{t} \omega\right\|_{L^{2}}^{p}+(\varphi(\nabla \omega), \kappa)-\frac{p n+4}{p+4-n}\|\nabla \omega\|_{L^{2}}^{2}+\lambda\left(\partial_{t} \omega, \omega\right)
$$

Let $\lambda, \alpha$ be small enough, one gets

$$
\begin{aligned}
\int g\left(|v|^{2}+\left|\omega_{0}\right|^{4}\right)|u|^{\frac{p n}{n-2}} d x & \leq \sigma\left(\|v\|_{L^{2}}^{\frac{p}{2}}+\left\|\omega_{0}\right\|_{p}^{4}\right)\|\omega\|_{H_{0}^{2}}^{\frac{p}{2}-1} \\
& \leq C\left(1+\|g\|_{L^{2}}^{n}\right)+\left\|\partial_{t} v\right\|_{L^{2}}^{n-2}+\left\|\nabla_{x} u\right\|_{L^{p}}^{n+1}+\|v\|_{L^{q}}^{\frac{n+1}{p}} \\
& \leq C\|v\|_{L^{p}}^{n+1-p}\left(\beta(u)^{\frac{p}{n}}+\left\|v_{0}\right\|_{L^{p}}^{p+1}\right) \beta(u)^{2+\frac{n}{q}}
\end{aligned}
$$

Where the constant $C_{\varepsilon}, \lambda_{\varepsilon}$ depend only on the $\varepsilon$.

Hence by the standard Galerkin method, interpolation inequality, Young's and Sobolev's inequality, this section can be estimated by

$$
\left(\varphi^{\prime}\left(\xi_{2}\right)-\varphi^{\prime}\left(\xi_{1}\right)\right)\left(\xi_{2}-\xi_{1}\right) \leq \theta\left(\left|\xi_{2}\right|-\left|\xi_{1}\right|\right)^{p}\left|\xi_{2}-\xi_{1}\right|^{2}, \quad \forall \xi_{1}, \xi_{2} \in R^{3}
$$

Noting that the energy solution of problem (1.1) is unique and the Lipschitz continuity in a weak space, There holds (2.6) and (2.7).

\section{Acknowledgements}

This work was supported by the Ph.D. Foundation of Henan University of Engineering (No. D2010012).

\section{References}

[1] A. V. Lair and A. W. Shaker, Large solutions of semi-linear elliptic problems, Nonlinear Anal. 37(6), 805-812, (2013).

[2] K.-S. Cheng and W.-M. Ni, on the structure of the conformal scalar curvature equation on RN, Indiana Univ. Math. J. 41(1), 261-278, (2012).

[3] Y.M. Qin, Shu-xian Deng. Decay Rate of Quasilinear Wave Equation with Viscosity. Acta Mathematicae Applicatae Sinica, 17(2014), 147-152.

[4] Nakao, M. Energy decay for the quasilinear wave equation with viscosity. Math. Z., 219: 289-299 (2015).

[5] T. Kato: Strong LP-solutions of the N-S equations in Rn, with applications to weak solutions. Math. Z. 187(2014), 471-480.

[6] V. Kalantarov, S. Zelik, Finite-dimensional attractors for the quasi-linear strongly-damped wave equation, J. Differential Equations (2013), 1-36. 